\title{
LEAST-SQUARES COLLOCATION WITH INTEGER PARAMETERS
}

\author{
P.J.G. Teunissen \\ Delft Institute for Earth Observation and Space Systems (DEOS) \\ Delft University of Technology \\ Kluyverweg 1 \\ 2629 HS Delft, The Netherlands \\ Fax: ++ 31152783711 \\ e-mail: P.J.G.Teunissen@tudelft.nl
}

\begin{abstract}
The prediction of spatially and/or temporal varying variates based on observations of these variates at some locations in space and/or instances in time, is an important topic in the various spatial and Earth sciences disciplines. This topic has been extensively studied, albeit under different names. In Geodesy it is generally referred to as least-squares collocation. The underlying model used is often of the trend-signal-noise type. This model is quite general and it encompasses many of the conceivable measurements. However, the methods of prediction based on these models have only been developed for the case the trend parameters are real-valued. In the present contribution we generalize the theory of least-squares collocation by permitting some or all of the trend parameters to be integer valued. We derive the solution of integer-based least-squares collocation and show how it compares to the solution of standard least-squares collocation.
\end{abstract}

Keywords: least-squares prediction, least-squares collocation, integer estimation, integerbased least-squares collocation

\section{INTRODUCTION}

The topic of this contribution is the prediction of spatially and/or temporal varying variates based on observations of these variates (or functionals thereof) at some locations in space and/or instances in time. This topic has been extensively studied, albeit under different names, in the various spatial and Earth sciences disciplines. In physical geodesy it is known as least-squares collocation (LSC). Fundamental contributions to this field have been made by [Krarup, 1969] and [Moritz, 1973], see also [Rummel, 1976], [Dermanis, 1980], [Sanso, 1986], [Grafarend and Rapp, 1980]. The underlying model of LSC is the so-called trend-signal-noise model. This model is quite general and it encompasses many of the conceivable geodetic measurements [Moritz, 1980, p. 111]. It also forms the basis of the concept of integrated geodesy as introduced in [Eeg and Krarup, 1973], see also [Krarup, 1980], [Hein, 1986].

LSC has been developed for models in which the trend parameters are real-valued. In the present contribution we will generalize the theory by permitting some or all of the trend parameters to be integer valued. Applications of such models can be found, for instance, in 
case of GNSS-based (GPS and/or Galileo) predictions of atmospheric fields (troposphere or ionosphere) or in case of InSAR-based predictions of deformation fields, see e.g. [Odijk, 2002], [Hanssen et al., 2001].

\section{LEAST-SQUARES PREDICTION}

In this contribution, we speak of estimation if a function of an observable random vector $y$ is used to guess the value of an unknown deterministic parameter vector $x$. If the function is given as $f$, then $f(y)$ is said to be the estimator of $x$ (we call it an estimate of $x$ if the function is taken of an outcome of $y$ ). We speak of prediction, if a function of an observable random vector $y$ is used to guess the outcome of another random, but unobservable, vector $y_{0}$. If the function is given as $g$, then $g(y)$ is said to be the predictor of $y_{0}$ (we call it a prediction of $y_{0}$ if the function is taken of an outcome of $y$ ). In the following, we assume the dispersion of $y$ and $y_{0}$ to be known, and their expectations (possibly unknown) to be linearly related to each other. Consider therefore the partitioned linear system of equations

$$
\left[\begin{array}{c}
y \\
y_{0}
\end{array}\right]=\left[\begin{array}{c}
A \\
A_{0}
\end{array}\right] x+\left[\begin{array}{c}
e \\
e_{0}
\end{array}\right]
$$

with matrices $A$ and $A_{0}$ of order $m \times n$ and $m_{0} \times n$, respectively, $x$ a nonrandom parameter vector and $\left[e^{T}, e_{0}^{T}\right]^{T}$ a random vector, with expectation and dispersion given as,

$$
E\left[\begin{array}{c}
e \\
e_{0}
\end{array}\right]=\left[\begin{array}{l}
0 \\
0
\end{array}\right] \text { and } D\left[\begin{array}{c}
e \\
e_{0}
\end{array}\right]=D\left[\begin{array}{c}
y \\
y_{0}
\end{array}\right]=\left[\begin{array}{cc}
Q_{y y} & Q_{y y_{0}} \\
Q_{y_{0} y} & Q_{y_{0} y_{0}}
\end{array}\right]
$$

respectively. The matrices $A$ and $A_{0}$ are assumed known, with $A$ being of full column rank. Also the dispersion matrix is assumed known and to be positive definite. All the entries of the above vectors and matrices are assumed to be real-valued.

The objective function we will work with is given by the positive definite quadratic form,

$$
F\left(y, y_{0}, x\right)=\left[\begin{array}{c}
y-A x \\
y_{0}-A_{0} x
\end{array}\right]^{T}\left[\begin{array}{cc}
Q_{y y} & Q_{y y_{0}} \\
Q_{y_{0} y} & Q_{y_{0} y_{0}}
\end{array}\right]^{-1}\left[\begin{array}{c}
y-A x \\
y_{0}-A_{0} x
\end{array}\right]
$$

If $y$ and $y_{0}$ are observable and $x$ is unknown, then the unique $\hat{x}^{\prime}$ satisfying $F\left(y, y_{0}, \hat{x}^{\prime}\right) \leq$ $F\left(y, y_{0}, x\right)$, for all $x \in R^{n}$, is said to be the least-squares estimator of $x$ based on both $y$ and $y_{0}$. If $y$ is observable, $x$ is known and $y_{0}$ is unobservable, then the unique $\hat{y}_{0}^{\prime}$ satisfying $F\left(y, \hat{y}_{0}^{\prime}, x\right) \leq F\left(y, y_{0}, x\right)$, for all $y_{0} \in R^{m_{0}}$, is said to be the least-squares predictor of $y_{0}$. In the present contribution, we are interested in the case that is a combination of the previous two problems. We assume $y$ to be observable, $x$ to be unknown and $y_{0}$ to be unobservable. Then the unique pair $\hat{x}, \hat{y}_{0}$ satisfying $F\left(y, \hat{y}_{0}, \hat{x}\right) \leq F\left(y, y_{0}, x\right)$, for all $x \in R^{n}, y_{0} \in R^{m_{0}}$, is said to be the least-squares estimator-predictor pair of $x, y_{0}$.

To solve the latter problem, first note that the quadratic form in Eq.(3) can be written as a sum of two squares (see Corollary 1 of the Appendix),

$$
F\left(y, y_{0}, x\right)=\|y-A x\|_{Q_{y y}}^{2}+\left\|y_{0}-A_{0} x-Q_{y_{0} y} Q_{y y}^{-1}(y-A x)\right\|_{Q_{y_{0} y_{0} \mid y}}^{2}
$$

with $Q_{y_{0} y_{0} \mid y}=Q_{y_{0} y_{0}}-Q_{y_{0} y} Q_{y y}^{-1} Q_{y y_{0}}$ and where we used the shorthand notation $\|.\|_{M}^{2}=$ $(.)^{T} M^{-1}($.$) . From Eq.(4) it follows that the estimator-predictor pair, \hat{x}$ and $\hat{y}_{0}$, are given as,

$$
\begin{aligned}
& \hat{x}=\left(A^{T} Q_{y y}^{-1} A\right)^{-1} A^{T} Q_{y y}^{-1} y \\
& \hat{y}_{0}=A_{0} \hat{x}+Q_{y_{0} y} Q_{y y}^{-1}(y-A \hat{x})
\end{aligned}
$$


Since $\hat{x}, \hat{y}_{0}$ set the second positive term of Eq.(4) equal to zero, while $\hat{x}$ minimizes the first positive term, it follows that Eq.(5) is indeed the solution to the minimization of the quadratic form of Eq.(4). It can be shown (see e.g. [Koch, 1980, p. 147], [Teunissen et al., 2005, p. 197]) that the simultaneously derived least-squares estimator-predictor pair $\hat{x}, \hat{y}_{0}$ constitute the BLUE (best linear unbiased estimator) and the BLUP (best linear unbiased predictor) of $x$ and $y_{0}$, respectively. This is a consequence of having used the inverse variance matrix of $\left(y^{T}, y_{0}^{T}\right)^{T}$ as weight matrix in Eq.(3). This choice will also be used for the mixed linear model with integer parameters (see Section 3). As a result the derived integer estimator can be shown to have the largest possible probability of correct integer estimation.

The following additional remarks can be made with respect to Eq.(5). ( $i)$ if $x$ would be known, then the least-squares predictor $\hat{y}_{0}^{\prime}$ can be obtained by replacing $\hat{x}$ in the expression for $\hat{y}_{0}$ by the known $x$. (ii) since $F\left(y, \hat{y}_{0}, \hat{x}\right) \leq F\left(y, \hat{y}_{0}, x\right)$, for all $x \in R^{n}$, it follows that the least-squares estimator of $x$ remains unaffected when $\hat{y}_{0}$ would be taken as if it were the observed $y_{0}$. This is also what one would expect, since $\hat{y}_{0}$ should not contain information about $x$ which is not already present in $y$. (iii) the first term in the expression of the least-squares predictor $\hat{y}_{0}, A_{0} \hat{x}$, is the least-squares estimator of $E\left(y_{0}\right)$, the mean of $y_{0}$. Thus if $y_{0}$ and $y$ are uncorrelated $\left(Q_{y_{0} y}=0\right)$, then the predictor of $y_{0}$ coincides with the estimator of $E\left(y_{0}\right)$. $(i v)$ if $y_{0}=y$ and thus $A_{0}=A$ and $Q_{y_{0} y}=Q_{y y}$, then $\hat{y}_{0}=y$. This shows that an observable is its own least-squares predictor.

\section{LEAST-SQUARES COLLOCATION}

An important case of the model in Eqs. (1) and (2), and one which has found wide-spread application in the spatial and Earth sciences, is the so-called trend-signal-noise model, see e.g. [Moritz, 1980]. It forms the basis of least-squares collocation. The model is applicable to a wide range of applications for which heterogeneous, over- as well as under-determined data need to be combined, see e.g. [Dermanis, 1980], [Rummel, 1976], [Sanso, 1986], [Grafarend and Rapp, 1980]. Such applications can be found in e.g. physical geodesy, mining engineering, hydrogeology, spatial data analysis, environmental engineering and digital image processing. The model also forms the basis of the concept of integrated geodesy as introduced in [Eeg and Krarup, 1973], see also [Krarup, 1980] and [Hein, 1986].

In the trend-signal-noise model the observable vector $y$ is written as a sum of three terms, $y=t+s+n$, with $t$ a deterministic, but unknown trend, $s$ a zero-mean random signal vector, and $n$ a zero-mean random noise vector. The trend is usually further parametrized in terms of an unknown parameter vector $x$ as $t=A x$. The signal and noise vector are assumed to be uncorrelated and their variance matrices are given as $Q_{s s}$ and $Q_{n n}$, respectively. Thus we have $y=A x+s+n$, with $Q_{y y}=Q_{s s}+Q_{n n}$ and where the sum of signal and noise, $s+n$, plays the role of the zero-mean random vector $e$ of Eq.(1). We may now apply Eq.(5) to separate trend, signal and noise. This gives

$$
\begin{aligned}
\hat{x} & =\left(A^{T}\left(Q_{s s}+Q_{n n}\right)^{-1} A\right)^{-1} A^{T}\left(Q_{s s}+Q_{n n}\right)^{-1} y \\
\hat{s} & =Q_{s s}\left(Q_{s s}+Q_{n n}\right)^{-1}(y-A \hat{x}) \\
\hat{n} & =Q_{n n}\left(Q_{s s}+Q_{n n}\right)^{-1}(y-A \hat{x})
\end{aligned}
$$

The first equation follows directly from applying the first equation of Eq.(5). The second and third equation are obtained from applying the second equation of Eq.(5), by interpreting $s$ and $n$, respectively, as the unobservable $y_{0}$. Thus $y_{0} \rightarrow\left(s^{T}, n^{T}\right)^{T}, A_{0} \rightarrow 0, e_{0} \rightarrow\left(s^{T}, n^{T}\right)^{T}$ and $e \rightarrow s+n$. Note that $y=A \hat{x}+\hat{s}+\hat{n}$, which reflects the property that the observable is its own predictor. Also note that $\hat{e}=\hat{s}+\hat{n}=y-A \hat{x}$ is the predictor of $e$. 
Often one can extend the trend-signal-noise model so as to hold true for an unobservable vector $y_{0}$ as well. This gives $y_{0}=A_{0} x+s_{0}+n_{0}$, in which $s_{0}$ and $n_{0}$ are uncorrelated zeromean random vectors, with variance matrices $Q_{s_{0} s_{0}}$ and $Q_{n_{0} n_{0}}$, respectively. The two signal vectors, $s_{0}$ and $s$, are assumed correlated $\left(Q_{s_{0} s} \neq 0\right)$, whereas the two noise vectors, $n_{0}$ and $n$, are (usually) assumed to be uncorrelated $\left(Q_{n_{0} n}=0\right)$. The signal plus noise vector of the unobservable vector plays now the role of the zero-mean random vector $e_{0}$ of Eq.(1). We may now apply Eq.(5) again to predict $y_{0}, s_{0}$ and $n_{0}$. In this case we set $y_{0} \rightarrow\left(y_{0}^{T}, s_{0}^{T}, n_{0}^{T}\right)^{T}$, $A_{0} \rightarrow\left(A_{0}^{T}, 0,0\right)^{T}, e_{0} \rightarrow\left(\left(s_{0}+n_{0}\right)^{T}, s_{0}^{T}, n_{0}^{T}\right)^{T}$ and $e \rightarrow s+n$. This gives

$$
\begin{aligned}
& \hat{y}_{0}=A_{0} \hat{x}+Q_{s_{0} s}\left(Q_{s s}+Q_{n n}\right)^{-1}(y-A \hat{x}) \\
& \hat{s}_{0}=Q_{s_{0} s}\left(Q_{s s}+Q_{n n}\right)^{-1}(y-A \hat{x}) \\
& \hat{n}_{0}=0
\end{aligned}
$$

Note that the predictor of the trend plus signal, $A_{0} x+s_{0}$, is identical to the predictor of $y_{0}$. Both are given as $A_{0} \hat{x}+\hat{s}_{0}$. In general, this is not the case. In the present situation, the two predictors coincide since the noise vector $n_{0}$ was assumed to be uncorrelated with $s$ and $n$. For the same reason, the predictor of $n_{0}$ is identically zero. The set of solutions (6) and (7) constitute the well-known least-squares collocation results.

\section{INTEGER-BASED LEAST-SQUARES PREDICTION}

We now extend the model of the previous section so as to include the option that some or all the parameters of $x$ are integer valued. We therefore assume $x=\left(x_{1}^{T}, x_{2}^{T}\right)^{T} \in Z^{p} \times R^{n-p}$. Thus the first $p$ entries of $x$ are assumed to be unknown integers and the last $n-p$ entries are assumed to be unknown real-valued parameters. The matrices $A, A_{0}$ are partitioned accordingly, $A=\left[A_{1}, A_{2}\right]$, $A_{0}=\left[A_{01}, A_{02}\right]$. We consider the same objective function $F\left(y, y_{0}, x\right)$, cf. Eq.(3), but with the stipulation that $x \in Z^{p} \times R^{n-p}$. Then the unique pair $\check{x}, \check{y}_{0}$ satisfying $F\left(y, \check{y}_{0}, \check{x}\right) \leq F\left(y, y_{0}, x\right)$, for all $x \in Z^{p} \times R^{n-p}, y_{0} \in R^{m_{0}}$, is said to be the integer-based least-squares estimator-predictor pair of $x, y_{0}$.

In order to determine this pair, we again decompose the objective function $F\left(y, y_{0}, x\right)$ into a sum of squares. This time however, it will be decomposed into a constant term and three variable terms. We have

$$
\begin{aligned}
F\left(y, y_{0}, x\right) & =\|y-A x\|_{Q_{y y}}^{2}+\left\|y_{0}-A_{0} x-Q_{y_{0} y} Q_{y y}^{-1}(y-A x)\right\|_{Q_{y_{0} y_{0} \mid y}}^{2} \\
& =\|y-A \hat{x}\|_{Q_{y y}}^{2}+\|\hat{x}-x\|_{Q_{\hat{x} \hat{x}}}^{2}+\left\|y_{0}-A_{0} x-Q_{y_{0} y} Q_{y y}^{-1}(y-A x)\right\|_{Q_{y_{0} y_{0} \mid y}}^{2} \\
& =\|y-A \hat{x}\|_{Q_{y y}}^{2}+\left\|\hat{x}_{1}-x_{1}\right\|_{Q_{\hat{x}_{1} \hat{x}_{1}}}^{2}+\left\|\hat{x}_{2}-x_{2}-Q_{\hat{x}_{2} \hat{x}_{1}} Q_{\hat{x}_{1} \hat{x}_{1}}^{-1}\left(\hat{x}_{1}-x_{1}\right)\right\|_{Q_{\hat{x}_{2} \hat{x}_{2} \mid \hat{x}_{1}}}^{2}+ \\
& \quad+\left\|y_{0}-A_{0} x-Q_{y_{0} y} Q_{y y}^{-1}(y-A x)\right\|_{Q_{y_{0} y_{0} \mid y}}^{2}
\end{aligned}
$$

with $\hat{x}=\left(\hat{x}_{1}^{T}, \hat{x}_{2}^{T}\right)^{T}$ given by the first equation of Eq.(5) and $Q_{\hat{x}_{2} \hat{x}_{2} \mid \hat{x}_{1}}=Q_{\hat{x}_{2} \hat{x}_{2}}-Q_{\hat{x}_{2} \hat{x}_{1}} Q_{\hat{x}_{1} \hat{x}_{1}}^{-1} Q_{\hat{x}_{1} \hat{x}_{2}}$. The first equality of Eq.(8) is Eq.(4). With $\|y-A x\|_{Q_{y y}}^{2}=\|y-A \hat{x}\|_{Q_{y y}}^{2}+\|\hat{x}-x\|_{Q_{\hat{x} \hat{x}}}^{2}$, which holds true since $A^{T} Q_{y y}^{-1}(y-A \hat{x})=0$ and $Q_{\hat{x} \hat{x}}=\left(A^{T} Q_{y y}^{-1} A\right)^{-1}$, the second equality of Eq.(8) follows from its first. The third equality follows then from the second since $\|\hat{x}-x\|_{Q_{\hat{x} \hat{x}}}^{2}=$ $\left\|\hat{x}_{1}-x_{1}\right\|_{Q_{\hat{x}_{1} \hat{x}_{1}}}^{2}+\left\|\hat{x}_{2}-x_{2}-Q_{\hat{x}_{2} \hat{x}_{1}} Q_{\hat{x}_{1} \hat{x}_{1}}^{-1}\left(\hat{x}_{1}-x_{1}\right)\right\|_{Q_{\hat{x}_{2} \hat{x}_{2} \mid \hat{x}_{1}}}^{2}$, the proof of which follows again from an application of Corollary 1. 
Note that the last term in the third decomposition of Eq.(8) can be made zero for any $x \in Z^{p} \times R^{n-p}$ and that the before last term can be made zero for any $x_{1} \in Z^{p}$. Hence, the solution for $\breve{x}, \check{y}_{0}$ follows as

$$
\begin{aligned}
& \check{x}_{1}=\arg \min _{z \in Z^{p}}\left\|\hat{x}_{1}-z\right\|_{Q_{\hat{x}_{1} \hat{x}_{1}}}^{2} \\
& \check{x}_{2}=\hat{x}_{2}-Q_{\hat{x}_{2} \hat{x}_{1}} Q_{\hat{x}_{1}}^{-1} \hat{x}_{1}\left(\hat{x}_{1}-\check{x}_{1}\right) \\
& \check{y}_{0}=A_{0} \check{x}+Q_{y_{0} y} Q_{y y}^{1}(y-A \check{x})
\end{aligned}
$$

Note that the structure of the predictor $\check{y}_{0}$ is identical to that of the predictor $\hat{y}_{0}$, cf. Eq.(5). That is, $\check{y}_{0}$ can be obtained from the expression of $\hat{y}_{0}$ by replacing $\hat{x}$ by $\check{x}$. Also note that $\check{x}_{2}$ can alternatively be expressed as $\check{x}_{2}=\left(A_{2}^{T} Q_{y y}^{-1} A_{2}\right)^{-1} A_{2}^{T} Q_{y y}^{-1}\left(y-A_{1} \check{x}_{1}\right)$. The steps in computing $\check{y}_{0}$ can now be summarized as follows. First one computes the least-squares estimate $\hat{x}=\left[\hat{x}_{1}^{T}, \hat{x}_{2}^{T}\right]^{T}$ based on the first expression of Eq.(5). Then $\hat{x}_{1}$ is used to determine the integer least-squares estimate $\check{x}_{1}$, which is the integer minimizer of $\left\|\hat{x}_{1}-z\right\|_{Q_{\hat{x}_{1} \hat{x}_{1}}}^{2}$. Finally, both $\check{x}_{1}$ and $\check{x}_{2}$ are used to compte $\check{y}_{0}$. Note, in case all entries of $x$ are integer valued, that the second line of Eq.(9) is absent and the first line gets replaced by $\check{x}=\arg \min _{z \in Z^{n}}\|\hat{x}-z\|_{Q_{\hat{x} \hat{x}}}^{2}$.

To see Eq.(9) at work, consider the following example.

Example 1 (prediction of error components) Consider the single equation

$$
y=a x+e_{1}+\ldots+e_{q}
$$

with scalar $a$ given, $x$ an unknown integer and the $e_{i}$ being $q$ uncorrelated, zero-mean random variables. If the sum $e=\sum_{i=1}^{q} e_{i}$ constitutes the total measurement error, then the $e_{i}$ may be considered the individual error components. The variance of $e_{i}$ will be denoted as $\sigma_{i}^{2}$. We will now predict such an individual error component using Eq.(9). Note that no real-valued parameters occur in the above model. Hence, only the first and the third expression of Eq.(9) need to be applied. Setting $x_{1} \rightarrow x, A \rightarrow a, y_{0} \rightarrow\left(e_{1}, \ldots, e_{q}\right)^{T}, A_{0} \rightarrow 0, e \rightarrow e_{1}+\ldots+e_{q}$ and $e_{0} \rightarrow\left(e_{1}, \ldots, e_{q}\right)^{T}$, yields $Q_{y y}=Q_{e e}=\sum_{j=1}^{q} \sigma_{j}^{2}, Q_{y_{0} y}=Q_{e_{0} e}=\left(\sigma_{1}^{2}, \ldots, \sigma_{q}^{2}\right)^{T},\left(\check{e}_{1}, \ldots, \check{e}_{q}\right)^{T}=$ $Q_{y_{0} y} Q_{y y}^{-1}(y-a \check{x})=\left(\sigma_{1}^{2}, \ldots, \sigma_{q}^{2}\right)^{T}\left(\sum_{j=1}^{q} \sigma_{j}^{2}\right)^{-1}(y-a \check{x})$, and thus

$$
\check{e}_{i}=\frac{\sigma_{i}^{2}}{\sum_{j=1}^{q} \sigma_{j}^{2}}(y-a \check{x})
$$

with the integer least-squares estimator of $x$ given as $\check{x}=[y / a]$, in which '[.]' denotes rounding to the nearest integer. This result shows that fractions of the residual $y-a \check{x}$ are assigned as predictors of the individual error components. The fractions are the ratios of the variance of the individual error component and the total variance. The predictors get an equal share of the residual when all variances are equal.

\section{LEAST-SQUARES COLLOCATION WITH INTEGER PARAMETERS}

We are now in the position to apply the results of the previous section to the trend-signal-noise model. For the separation of trend, signal and noise, this gives

$$
\begin{aligned}
\check{x}_{1} & =\arg \min _{z \in Z^{p}}\left\|\hat{x}_{1}-z\right\|_{Q_{\hat{x}_{1} \hat{x}_{1}}}^{2} \\
\check{x}_{2} & =\hat{x}_{2}-Q_{\hat{x}_{2} \hat{x}_{1}} Q_{\hat{x}_{1} \hat{x}_{1}}^{-1}\left(\hat{x}_{1}-\check{x}_{1}\right) \\
\check{s} & =Q_{s s}\left(Q_{s s}+Q_{n n}\right)^{-1}(y-A \check{x}) \\
\check{n} & =Q_{n n}\left(Q_{s s}+Q_{n n}\right)^{-1}(y-A \check{x})
\end{aligned}
$$




\section{4}

where $\hat{x}$ is given as

$$
\hat{x}=\left(A^{T}\left(Q_{s s}+Q_{n n}\right)^{-1} A\right)^{-1} A^{T}\left(Q_{s s}+Q_{n n}\right)^{-1} y
$$

If we compare this result with standard least-squares collocation, we note that an additional step is included, namely that of computing $\check{x}=\left(\check{x}_{1}^{T}, \check{x}_{2}^{T}\right)^{T}$. If we extend the trend-signal-noise model so as to hold true for an unobservable vector $y_{0}=A_{0} x+s_{0}+n_{0}$ as well, we obtain the integer-based least-squares collocation result as

$$
\begin{aligned}
& \check{y}_{0}=A_{0} \hat{x}+Q_{s_{0} s}\left(Q_{s s}+Q_{n n}\right)^{-1}(y-A \check{x}) \\
& \check{s}_{0}=Q_{s_{0} s}\left(Q_{s s}+Q_{n n}\right)^{-1}(y-A \check{x}) \\
& \check{n}_{0}=0
\end{aligned}
$$

Compare this result with (7). The overall conclusion is thus reached, that the solution of integer-based collocation has the same structure of standard least-squares collocation, be it that an additional step needs to be included to take care of the integer estimation of the trend parameters. We conclude with an example.

Example 2 (ionospheric prediction) Consider as a trend-signal-noise model, the single-frequency, single epoch, geometry-free GPS equations, based on double-differenced (DD) carrier phase and pseudorange,

$$
\begin{array}{rrr}
y_{1}= & \lambda x_{1}+x_{2}+s+n_{1} \\
y_{2}= & & +x_{2}-s+n_{2}
\end{array}
$$

with $x_{1}$ the unknown integer DD carrier phase ambiguity, $\lambda$ the known wavelength of the carrier phase, $x_{2}$ the unknown DD range, $s$ the residual ionospheric signal, and $n_{1}$ and $n_{2}$ the noise of the carrier phase and the pseudorange, respectively. Let $\sigma_{1}^{2}$ and $\sigma_{2}^{2}$ denote the variances of the DD carrier phase and pseudorange, respectively, and let $\sigma_{s}^{2}$ denote the variance of the ionospheric signal. Then

$$
\left[\begin{array}{l}
\hat{x}_{1} \\
\hat{x}_{2}
\end{array}\right]=\left[\begin{array}{c}
\left(y_{1}-y_{2}\right) / \lambda \\
y_{2}
\end{array}\right] \text { and } Q_{\hat{x} \hat{x}}=\frac{1}{\lambda^{2}}\left[\begin{array}{cc}
4 \sigma_{s}^{2}+\sigma_{1}^{2}+\sigma_{2}^{2} & -\lambda\left(2 \sigma_{s}^{2}+\sigma_{2}^{2}\right) \\
-\lambda\left(2 \sigma_{s}^{2}+\sigma_{2}^{2}\right) & \lambda^{2}\left(\sigma_{s}^{2}+\sigma_{2}^{2}\right)
\end{array}\right]
$$

from which the integer-based least-squares parameter solution follows as,

$$
\begin{aligned}
& \check{x}_{1}=\left[\hat{x}_{1}\right] \\
& \check{x}_{2}=\hat{x}_{2}+\lambda \frac{2 \sigma_{s}^{2}+\sigma_{2}^{2}}{4 \sigma_{s}^{2}+\sigma_{1}^{2}+\sigma_{2}^{2}}\left(\hat{x}_{1}-\check{x}_{1}\right)
\end{aligned}
$$

If we want to predict the signal $s_{0}$ (e.g. the residual ionospheric delay at another time instant), then $s_{0}$ plays the role of $y_{0}$ and the integer-based least-squares predictor $\check{s}_{0}=Q_{s_{0} y} Q_{y y}^{-1}(y-A \check{x})$ works out as

$$
\check{s}_{0}=\frac{\sigma_{s_{0} s} / \sigma_{1}^{2}}{1+\sigma_{s}^{2} / \sigma_{1}^{2}+\sigma_{s}^{2} / \sigma_{2}^{2}}\left[\left(y_{1}-\lambda \check{x}_{1}-\check{x}_{2}\right)-\frac{\sigma_{1}^{2}}{\sigma_{2}^{2}}\left(y_{2}-\check{x}_{2}\right)\right]
$$




\section{REFERENCES}

Dermanis, A. (1980): Adjustment of geodetic observations in the presence of signals. In: Proceedings of the International School of Advanced Geodesy. Bollettino di Geodesia e Scienze Affini. Vol. 38, pp. 419-445.

Eeg, J. and T. Krarup (1973): Integrated geodesy. Dan. Geod. Inst., int. rep. 7, Copenhagen.

Grafarend, E.W. (1976): Geodetic applications of stochastic processes. Phys. Earth Planet. Interiors, 12, 151-179.

Grafarend, E.W. and R. H. Rapp (Eds.)(1980): Advances in geodesy. Selected papers from Rev. Geophys. Space Phys., Richmond, Virg., Am. Geophys. Union, Washington.

Hanssen, R.F., P.J.G. Teunissen, P. Joosten (2001): Phase ambiguity resolution for stacked radar interferometric data. In: Proc. KIS2001, International Symposium on Kinematic Systems in Geodesy, Geomatics and Navigation, Banff, Canada, pp. 317-320.

Hein, G.W. (1986): Integrated geodesy. In: Mathematical and numerical techniques in physical geodesy, H. Suenkel (Ed.), Lecture Notes in Earth Sciences, Springer Verlag, No. 7, pp. 505-548.

Koch, K.R. (1980): Parameterschaetzung und Hypothesentests in linearen Modellen, Dummler, Bonn.

Krarup, T. (1969): A contribution to the mathematical foundation of physical geodesy. Publ. Danish Geod. Inst. 44, Copenhagen.

Krarup, T. (1980): Integrated geodesy. In: Proceedings of the International School of Advanced Geodesy. Bollettino di Geodesia e Scienze Affini. Vol. 38, pp. 480-496.

Moritz, H. (1973): Least-squares collocation. DGK, A 59, Muenchen.

Moritz, H. (1980): Advanced Physical Geodesy. Herbert Wichmann Verlag Karlsruhe.

Sanso, F. (1986): Statistical methods in physical geodesy. In: Mathematical and numerical techniques in physical geodesy, H. Suenkel Ed., Lecture Notes in Earth Sciences, SpringerVerlag, Vol. 7, 49-156.

Rummel, R. (1976): A model comparison in least-squares collocation. Bull. Geod., 50, 181192.

Teunissen, P.J.G., D.G. Simons and C.C.J.M. Tiberius (2005): Probability and Observation Theory. Lecture Notes Delft University of Technology, 364 p.

Tscherning, C.C. (1978): Collocation and least-squares methods as a tool for handling gravity field dependent data obtained through space research techniques. Bull. Geod., 52, 199212. 


\section{6}

\section{APPENDIX}

\section{Corollary 1}

A quadratic form

$$
F=\left[\begin{array}{l}
a \\
b
\end{array}\right]^{T}\left[\begin{array}{ll}
Q_{a a} & Q_{a b} \\
Q_{b a} & Q_{b b}
\end{array}\right]^{-1}\left[\begin{array}{l}
a \\
b
\end{array}\right]
$$

can be decomposed into a sum of two quadratic forms as

$$
F=a^{T} Q_{a a}^{-1} a+\left(b-Q_{b a} Q_{a a}^{-1} a\right)^{T}\left(Q_{b b}-Q_{b a} Q_{a a}^{-1} Q_{a b}\right)^{-1}\left(b-Q_{b a} Q_{a a}^{-1} a\right)
$$

Proof The following block triangular factorization is easily verified,

$$
\left[\begin{array}{ll}
Q_{a a} & Q_{a b} \\
Q_{b a} & Q_{b b}
\end{array}\right]=\left[\begin{array}{cc}
I & 0 \\
Q_{b a} Q_{a a}^{-1} & I
\end{array}\right]\left[\begin{array}{cc}
Q_{a a} & 0 \\
0 & Q_{b b}-Q_{b a} Q_{a a}^{-1} Q_{a b}
\end{array}\right]\left[\begin{array}{cc}
I & 0 \\
Q_{b a} Q_{a a}^{-1} & I
\end{array}\right]^{T}
$$

The inverse of this matrix follows, with

$$
\left[\begin{array}{cc}
I & 0 \\
Q_{b a} Q_{a a}^{-1} & I
\end{array}\right]^{-1}=\left[\begin{array}{cc}
I & 0 \\
-Q_{b a} Q_{a a}^{-1} & I
\end{array}\right]
$$

as

$$
\left[\begin{array}{ll}
Q_{a a} & Q_{a b} \\
Q_{b a} & Q_{b b}
\end{array}\right]^{-1}=\left[\begin{array}{cc}
I & 0 \\
-Q_{b a} Q_{a a}^{-1} & I
\end{array}\right]^{T}\left[\begin{array}{cc}
Q_{a a}^{-1} & 0 \\
0 & \left(Q_{b b}-Q_{b a} Q_{a a}^{-1} Q_{a b}\right)^{-1}
\end{array}\right]\left[\begin{array}{cc}
I & 0 \\
-Q_{b a} Q_{a a}^{-1} & I
\end{array}\right]
$$

Substitution of this expression into the quadratic form $F$ of Eq.(12) gives the desired decomposition.

End of Proof

Received: 2006-09-05,

Reviewed: 2006-09-13, by W. Pachelski,

Accepted: 2006-09-13. 